\title{
Efectos farmacológicos de los Cannabinoides
}

\author{
LeZA, J.C.; LoRenzo, P.
}

Departamento de Farmacología. Facultad de Medicina. Universidad Complutense de Madrid.

Dirigir correspondencia: Prof. J.C. Leza. Dpto. de Farmacología. Facultad de Medicina. Universidad Complutense de Madrid. 28040 Madrid. Tel./fax 913941463.

\section{Resumen}

Se resumen los efectos farmacológicos in vivo de los cannabinoides. Aunque la mayor parte del conocimiento actual sobre los mecanismos de acción de los cannabinoides se ha desarrollado en modelos animales, los efectos finales de estos compuestos en el humano difieren de los efectos que pueden observarse en animales, en especial los conductuales y psicológicos. Los cannabinoides presentes en el humo de la marihuana provocan un amplio rango de efectos somáticos en el humano, en el aparato cardiovascular (taquicardias supraventriculares y alteraciones en el ECG), aparato respiratorio (efectos irritativos, a pesar de las propiedades broncodilatadoras de los cannabinoides), y en el ojo (efectos irritativos debidos al humo y disminución de la presión intraocular). La exposición crónica produce además, alteraciones endocrinológicas y metabólicas. Los efectos conductuales de estas drogas en el humano varían en función del estado previo del sujeto y de sus expectativas, y van desde la euforia y sensación de bienestar hasta el desarrollo de patologías psiquiátricas.

Palabras clave: animal, humano, cannabinoides, efectos clínicos.

\section{Summary}

It is summarised the current knowledge about the in vivo pharmacological effects of cannabinoids. Although much of the recent progress in understanding the mechanisms of action of cannabinoids has derived from the animal models of addiction, the effects of these drugs in humans differs from those in animals, specially behavioural and psychotropic effects. Cannabinoid components of marijuana smoke are known to exert a broad range of somatic effects in humans in cardiovascular system (supraventricular tachycardias and ECG changes), respiratory system (irritative effects, in spite of the bronchodilatatory properties of cannabinoids), eye (irritative effects directly due to the smoke and decrease in intraocular pressure). Chronic exposure leads to endocrine and metabolic changes. The behavioral effects of these drugs in humans differ upon the previous status and expectations of the smoker, ranging from euphoria and a feeling of well-being to psychiatric disorders.

Key words: animal, humans, cannabinoids, clinical effects.

\section{INTRODUCCIÓN}

E s este uno de los capítulos de la Farmacología que mayor atención han recibido en la última década (rev. en Gold, 1991; Adams y Martin, 1996). Tras el descubrimiento de los receptores CB y del mecanismo de acción de los ligandos endógenos, se trata ahora de entender cuáles son los los mecanismos por los que se producen los efectos farmacológicos conocidos desde hace tiempo y explicar nuevos efectos, algunos de ellos con posible indicación terapéutica. Este esfuerzo está dirigido al diseño de moléculas que puedan separar los efectos psicoactivos de los posibles efectos beneficiosos. 
A la hora de exponer de manera clara los efectos farmacológicos de los cannabinoides nos encontramos ante un problema de difícil solución, por cuanto los experimentos en animales de experimentación no nos proporcionan datos absolutamente extrapolables a la especie humana, no sólo en lo que se refiere a los efectos en el comportamiento, sino también a los efectos en diferentes órganos y sistemas. Además, las preparaciones de Cannabis utilizadas por el humano son mezclas de muchos compuestos cuyos efectos a veces se contraponen. Al final de este capítulo expondremos los efectos de los cannabinoides presentes en el cigarrillo de marihuana, la vía de administración más común en nuestro medio.

\section{EFECTOS FARMACOLÓGICOS DE LOS CANNABINOIDES:}

\subsection{Efectos conductuales.}

Los efectos conductuales de la inhalación de humo del Cannabis en humanos son realmente complejos y dependen de muchas variables (la personalidad del consumidor, el ambiente de consumo, las expectativas, etc...). En general puede decirse que los efectos conductuales de estos compuestos a dosis bajas son de tipo depresor, pero que se convierten en excitatorios tras estímulos mínimos, y que a dosis altas, los efectos predominantes son claramente de tipo depresor. Los efectos conductuales de dosis bajas de cannabinoides en roedores han sido denominados eufemísticamente "en palomitas de maíz": Ios animales se encuentran deprimidos, pero si alguno de ellos es estimulado con un leve ruido o movimiento, se produce una hiperreflexia que hace que el ratón salte en su jaula. Curiosamente, si este animal al caer, toca algún otro, éste saltará de nuevo, provocando en la jaula de ratones algo parecido a lo que vemos cuando los granos de maíz estallan al preparar palomitas (rev. en Pertwee, 1997). Evidentemente, sería arriesgado si asumiéramos que esto es lo que ocurre en la especie humana, pero este dato puede acercarnos más a la comprensión de los complejos efectos conductuales de los cannabinoides en humanos si nos preguntamos por qué en algunos sujetos el fumarse un porro tiene efectos sedantes cuando se fuma en soledad, y efectos contrarios cuando ese mismo sujeto está en un ambiente de hiperestimulación acústica o luminosa.

Este característico estado de hiperreflexia o hiperestimulación dentro de la fase depresora de los cannabinoides es especial entre todas las sustancias psicotropas utilizadas como drogas de abuso.

\subsection{Efectos sobre la agresividad.}

Los efectos de los cannabinoides sobre el comportamiento frente a otros individuos también muestran una dualidad agresividad/apatía. Los estudios realizados en animales demuestran que los extractos de marihuana o el propio $\Delta^{9}-\mathrm{THC}$ inducen un estado de agresividad. El estado de excitación y los impulsos irrefrenables que se observan en el humano ocurren tras ingestión o inhalación aguda de cannabinoides. Además, como se describe posteriormente, la megalomanía es un rasgo frecuente en la intoxicación leve. Todo esto puede condicionar un "conflicto de intereses" entre individuos consumidores o entre estos y otros no consumidores.

Sin embargo, aunque esto no ha podido ser comprobado en animales, se dispone de una considerable cantidad de información acerca del síndrome amotivacional en humanos consumidores crónicos de Cannabis (Quiroga, 2000), y resulta lógico pensar que esto pueda ocurrir bajo los efectos de sustancias depresoras del SNC.

\subsection{Efectos sobre la actividad locomoto-} ra.

Los cannabinoides pueden producir ciertas alteraciones de los movimientos tipo ataxia en animales grandes como el perro. Incluso 
se ha comprobado que puede desarrollarse catalepsia, además de potenciar la hipoquinesia inducida por reserpina en ratas. Efectos similares se han observado en humanos. Estos pueden ser resultado de la interacción de los cannabinoides con receptores CB localizados en cerebelo y en los ganglios basales, en particular en regiones que procesan comportamientos motores y regulan información sensorio-motora.

\subsection{Efectos sobre la temperatura corpo- ral.}

Como la mayoría de los depresores del SNC, los THC inducen hipotermia en animales normotérmicos. Además, también reducen la temperatura en animales sometidos a hipertermia inducida por inyección de toxinas de microorganismos, aunque los THC son más potentes en este último caso que en animales normotérmicos. También está demostrado que los efectos sobre la temperatura se alcanzan con dosis menores que las que producen efectos conductuales. Parece claro que interfieren en el centro hipotalámico regulador de la temperatura, aunque hasta el momento se desconoce el mecanismo íntimo de este efecto.

Se desarrolla tolerancia a estos efectos.

\subsection{Efectos sobre la memoria.}

La presencia de receptores en áreas cerebrales relacionadas con la memoria (fundamentalmente hipocampo) ha hecho que el estudio de las alteraciones en la memoria inducida por cannabinoides sea un área de máximo interés.

El THC dificulta el aprendizaje en experimentos llevados a cabo en roedores. En humanos pueden producir alteraciones cuantificables en test psicológicos, y a la dosis a que aparecen alteraciones en los tests de conducta, se comprueba que se origina confusión y dificultad de la memorización de tareas sencillas. Además, se produce inte- rrupción de la continuidad del discurso (lagunas) y lenguaje monótono.

Sin embargo, de entre todos los ligandos endógenos, hay uno en particular, el 2-araquidonilglicerol, identificado al principio en intestino, que se ha mostrado como un mediador del fenómeno de la potenciación neuronal perdurable ("long term potentiation, $L T P^{\prime \prime}$ ), un mecanismo esencial en los procesos de memoria.

\subsection{Efectos sobre la conducta alimenti- cia y la ingesta.}

Uno de los comentarios más frecuentes es que los consumidores de Cannabis presentan un ansia por comer dulces. Aparte de ciertas explicaciones desde el punto de vista psicológico (involución de la personalidad, regresión a la personalidad infantil), realmente esto no ha podido ser comprobado científicamente; de hecho, los datos más repetidos en experimentos de laboratorio apuntan a una disminución de la ingesta de comida en roedores. Por otra parte, el efecto antiemético pudiera contribuir en cierta medida al aumento de la ingestión de comida en algunos pacientes con cierto grado de anorexia.

\subsection{Efecto analgésico.}

Se trata de uno de los efectos mejor caracterizados de los cannabinoides. Desde los primeros estudios científicos sobre los THC se sabe que poseen actividad analgésica y, en general, que los metabolitos $11-\mathrm{OH}$ son más potentes que los compuestos iniciales. En particular, 9-nor-9 $\beta-\mathrm{OH}$-hexahidrocannabinol, levonantradol y su metabolito des-acetil-levonantradol son los más potentes. Además, se ha comprobado que los antagonistas de receptores $\mathrm{CB}_{1}$ producen hiperalgesia.

La acción antinociceptiva de los cannabinoides se establece a nivel espinal y supraespinal (la sección quirúrgica de las aferentes nociceptivas espinales -"espinalización"- sólo reduce en parte la acción analgésica), y no 
parece tener relación con mecanismos $\mu$ ó $\delta$ opioides, ya que ni naloxona $(\mu)$ ni ICl-174864

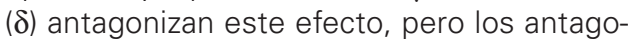
nistas k como norbinaltorfimina sí reducen la analgesia inducida por $\Delta^{9}$-THC. Sin embargo, se ha comprobado que dosis subefectivas de $\Delta^{9}$-THC potencian la analgesia inducida por morfina en algunos test de analgesia.

\subsection{Efecto antiemético.}

En humanos, la administración de $\Delta^{9}-\mathrm{THC}$ y de otros agonistas CB (Lorenzo y Leza, 2000) previene las náuseas y vómitos en pacientes en tratamiento con anticancerosos. Hasta este momento no se dispone de datos concluyentes que expliquen el mecanismo de acción antiemético.

\subsection{Efecto anticonvulsivante.}

Está bastante claro que los cannabinoides, en particular canabidiol (CBD) y $\Delta^{9}-\mathrm{THC}$, son activos en varios modelos animales de actividad anticonvulsivante. El principal metabolito del $\Delta^{9}-\mathrm{THC}, 11-\mathrm{OH}-\Delta^{9}-\mathrm{THC}$, y su análogo sintético, dimetilheptilpirano, son más potentes que los compuestos de origen. Este efecto parece ser debido a que los THC deprimen la transmisión neuronal cortical entre los dos hemisferios. Además, potencian la actividad anticonvulsivante de las benzodiacepinas. En realidad, CBD inhibe las convulsiones tónicas y clónicas inducidas por inhibidores GABA, ácido 3-mercaptopropiónico, picrotoxina, ácido isonicotínico y bicuculina, pero no bloquea las convulsiones inducidas por estricnina (un antagonista de la glicina). Estos datos demuestran que la acción anticonvulsivante de los THC está en relación más con una potenciación o facilitación de los mecanismos GABA que con mecanismos glicinérgicos.

\subsection{Efecto antiinflamatorio.}

Los datos sobre la capacidad antiinflamatoria de los THC son escasos, aunque se ha descrito una cierta actividad antiinflamatoria en tests de inyección de sustancias irritantes en pata de roedor.

\subsection{Efectos en el sistema inmune.}

Los cannabinoides suprimen las respuestas celulares y humorales in vivo e in vitro. Ratones tratados con THC muestran un aumento de la susceptibilidad a las bacterias gram negativas en relación con la supresión de la formación de anticuerpos, citoquinas y depresión de la actividad de las células NK. El grado de este efecto inmunosupresor varía con el tejido examinado. Los animales jóvenes parecen afectarse más que los adultos. No obstante, la experiencia clínica no ha demostrado que los consumidores de marihuana sean más susceptibles a las infecciones.

\subsection{Efectos en el sistema endocrino.}

Además de los efectos sobre la regulación hipotalámica de prolactina y gonadotropinas, que se presentan en el siguiente punto, los THC producen una estimulación potente de la liberación de ACTH y, secundariamente, de corticosteroides. Además, reducen la TRH y los niveles plasmáticos de $\mathrm{T}_{3} \mathrm{y}_{4}$, y disminuyen los niveles plasmáticos de $\mathrm{GH}$ y de PRL. Se desarrolla tolerancia a todos estos efectos.

\subsection{Efectos sobre la reproducción.}

En general, es un hecho aceptado que el $\Delta^{9}$-THC produce una disminución de la secreción de FSH, LH y prolactina. La capacidad del $\Delta^{9}$-THC de disminuír la liberación de LHRH del hipotálamo es revertida por naloxona, lo que indica que el sistema opioide endógeno está implicado en este efecto.

En animales macho, la administración crónica de $\Delta^{9}$-THC produce una disminución del peso corporal y del volumen de los testículos e involución de las células de Leydig en la mayoría de las especies, efecto que puede ser revertido por administración de andrógenos. 
En cuanto al comportamiento sexual, los estudios llevados a cabo en animales sugieren que el $\Delta^{9}$-THC aumenta el tiempo de latencia hasta la cópula y hasta la eyaculación, aunque no se disminuye el número total de cópulas. En humanos no se han podido demostrar estos efectos, más bien parece que el aumento del interés sexual está en relación con los cambios psicológicos y la desinhibición.

Algunos estudios revelan que la descendencia masculina de ratas madres expuestas a THC durante la gestación tienen fertilidad reducida y anormalidades testiculares, aunque esto no ha podido ser comprobado en humanos. Una posible explicación es que el $\Delta^{9}$-THC se comporta como un competidor leve de la unión de estradiol a sus receptores citoplasmáticos. De hecho, parece que el efecto de $\Delta^{9}$-THC es más el de un agonista parcial: dosis bajas producen efecto estrogénico (aumenta el peso y la vascularización uterina en ratas ovariectomizadas).

En mujeres, se ha comprobado que concentraciones bajas de cannabinoides, como las que se alcanzan con un cigarrillo de marihuana, pueden disminuír el pico de LH plasmática.

\subsection{Efectos sobre el aparato cardiovas- cular.}

Aunque los datos no son absolutamente concluyentes, parece que los efectos resultantes son bradicardia y disminución de la presión arterial en sujetos normotensos y también en animales con hipertensión inducida por estímulos ambientales - esto ha dado pie al ensayo de compuestos cannabinoides en el tratamiento de la hipertensión inducida por estrés-. Se desarrolla tolerancia al efecto hipotensor.

Los estudios llevados a cabo en animales no son consistentes; los datos más repetidos son bradicardia, disminución del volumen minuto y alteraciones en los parámetros ECG. Sin embargo, en monos y en humanos, el efecto predominante sobre la frecuencia cardiaca parece ser de rebote: taquicardia por disminución de la presión arterial. Incluso se produce un aumento transitorio de la presión arterial que desaparece a los pocos minutos.

\subsection{Efectos sobre el aparato respirato- rio.}

Aunque no se producen cambios constantes en la frecuencia respiratoria, durante mucho tiempo se ha asociado el uso de marihuana con bronquitis y asma, efecto que puede deberse a la irritación del humo del cigarrillo, ya que la respuesta aguda del THC es broncodilatadora y relativamente duradera, tanto en animales de experimentación como en humanos sanos o asmáticos (Tashlin y cols., 1980; Benson y Bentley, 1995).

Por otra parte, el hecho de fumar marihuana conlleva un aumento de la inhalación de sustancias cancerígenas (significativamente mayor que con tabaco, en el caso de benzopirenos y benzantracenos)

\subsection{Efecto sobre el ojo.}

Aparte del efecto irritante del humo del sujeto que está fumando o de la exposición cuando se está en ambientes cerrados, tanto los cannabinoides naturales como la anandamida disminuyen la presión intraocular e inducen hiperemia conjuntival. Se han realizado algunos estudios para evaluar el posible uso clínico de estos compuestos como antiglaucomatosos.

\subsection{Efectos sobre el crecimiento $y$ degeneración celular.}

Los numerosos trabajos que tratan de estudiar este punto no presentan resultados concluyentes hasta el momento (rev. en Marselos y Karamanakos, 1999). La exposición de cultivos celulares a concentraciones elevadas de cannabinoides pueden provocar roturas de cromosomas, delecciones, translocaciones o hipoploidia, pero se trata de estudios muy heterogéneos en cuanto a pro- 
tocolos de tratamiento, animales utilizados o estirpes celulares empleadas. Por lo tanto, este extremo no ha podido ser absolutamente confirmado.

Recientemente se ha informado de que la anandamida posee características de co-estimulador de la formación de células sanguíneas, juntamente con el factor de crecimiento hemopoyético.

\subsection{Efectos en la descendencia.}

Una de las líneas de investigación más interesantes en los últimos tiempos sobre los efectos de los cannabinoides son los efectos sobre la descendencia. Se han identificado áreas de localización específica de receptores CB en cerebros en desarrollo (cuerpo calloso, comisura anterior, estría terminal), algunas de las cuales desaparecen en el periodo adulto. En ratas se han determinado ciertas alteraciones del desarrollo hipofiso-pituitario (disminución de los niveles de gonadotropina, LH y FSH) y de los procesos de memoria y aprendizaje en la descendencia (Navarro y cols., 1995). También se ha identificado que incrementan la embrioletalidad y producen retraso en el crecimiento de las crías tras la exposición perinatal a marihuana.

Los efectos en la descendencia de mujeres que inhalaban humo de Cannabis durante en el embarazo no son concluyentes (García y Mur, 1991).

\section{ACCIONES DE LOS CANNABINOIDES EN EL HUMANO.}

Hasta ahora se han descrito sistemáticamente los efectos farmacológicos de los cannabinoides en los distintos órganos y sistemas. Describiremos ahora cuál es la resultante de todos estos efectos en el humano, es decir, cuál es la traducción clínica, aunque es necesario recordar que los efectos de los cannabinoides dependen de muchos factores, el primero de ellos es la expectativa del sujeto, incluso su habilidad para inhalar. Desde hace tiempo se sabe que los efectos psicológicos varían según el ambiente, siendo en general relajantes si el sujeto inhala solo y euforizantes si se fuma en grupo (Tennant y cols., 1971). Por otra parte, recordemos que estas sustancias se ingieren en general junto con otras drogas (tabaco, alcohol) que pueden interferir con sus efectos.

Pueden diferenciarse manifestaciones agudas y crónicas:

\subsection{Sintomatología aguda.}

-Sistema cardiovascular: se han observado taquicardias sinusales (120 - 140 lpm), aunque a dosis elevadas pueden producir bradicardia, hipotensión ortostática, alteraciones electrocardiográficas inespecíficas del segmento ST y la onda T y extrasístoles ventriculares.

-Aparato respiratorio: los cannabinoides producen broncodilatación, sobre todo en personas con cierto grado de broncoconstricción, aunque, por lo general, este efecto se ve enmascarado por el efecto irritante del humo: laringitis, traqueítis y bronquitis.

-Aparato digestivo: la administración de THC puede producir diarrea, aunque se supone que es debido a la contaminación por $E$ coli o Salmonella.

-Ojo: aunque la irritación de los ojos es el efecto característico, debido al humo, los cannabinoides producen dilatación de vasos conjuntivales y enrojecimiento ocular característico. En algunos casos puede evidenciarse ptosis y disminución de la presión intraocular.

-Efectos psicológicos: En cuanto a los efectos psíquicos subjetivos, estos se inician a los pocos minutos de la inhalación y duran 1- 1.5 h (si la administración es oral, se inician más tarde y duran más). En resumen, comienzan con un periodo excitatorio, con una sensación de euforia y bienestar. La percepción temporal está alterada, lo mismo con la percepción de la música y de los colores. 
En general no hay alteraciones importantes de la conducta, aunque puede existir ideación fija e impulsos irresistibles. No es rara una hilaridad, locuacidad y megalomanía, sobre todo si el consumo se hace en grupo. Es habitual que el episodio tóxico termine con un acceso de bulimia. También se ha demostrado una alteración de la memoria inmediata.

Tras la exposición aguda del compuesto aparece la segunda fase de la secuencia bifásica antes mencionada: un estado de relajación.

-Las funciones motoras complejas se ven también alteradas. Esto ha generado gran intranquilidad acerca del riesgo que implica conducir vehículos bajo los efectos de los derivados Cannabis, y hay estudios que demuestran que hay una alteración en la percepción de las distancias y un aumento del tiempo de reacción. Sin embargo, otros estudios no llegan a datos tan concluyentes, por lo que hasta la fecha no se puede establecer esta relación.

-Efectos psiquiátricos: se han descrito reacciones anormales en forma de crisis de ansiedad o ataques de pánico, de breve duración, o de accesos depresivos. Estos casos deben considerarse urgencias psiquiátricas y tratarse como tales (Giannini, 1991; Millman y cols., 1997). La intoxicación "normal" no implica riesgos especiales en una persona sana y no precisa tratamiento (ver también Quiroga, 2000).

-Otros efectos: sequedad de boca, sed y aumento del apetito (con una predilección especial por los dulces, sin que esto se haya podido relacionar con alteraciones en el metabolismo de los hidratos de carbono).

\subsection{Sintomatología crónica.}

-Aparato respiratorio: los efectos más comunes de la inhalación del humo de marihuana son del desarrollo de bronquitis, laringitis y asma. También se ha demostrado una alta incidencia de bronconeumopatías crónicas en individuos que fuman cannabinoides durante largos periodos de tiempo.
-Hormonas y reproducción: aunque en una primera fase estos compuestos aumentan el interés sexual, en fumadores crónicos se demuestra una disminución de la libido. En mujeres, ciclos anovulatorios, y en varones anomalías estructurales y/o en la movilidad de los espermatozoides y oligospermia.

-Los efectos en el feto expuesto a cannabinoides se exponen con más detalle en más adelante.

-Efectos psicológicos y psiquiátricos: los fumadores crónicos de estas sustancias pueden desarrollar cuadros de reacciones de pánico, impresiones retrospectivas, reacciones psicóticas, ansiedad, depresión y trastornos de la memoria inmediata. El llamado síndrome amotivacional se describe con más detalle en Quiroga (2.000).

\section{CONCLUSIONES}

Los efectos de la inhalación del humo del Cannabis son complejos y dependen de muchas variables (personalidad del consumidor, ambiente del consumo, expectativas del consumidor...). Tras la inhalación del humo del cigarrillo de marihuana se presenta una constelación de signos y síntomas que afectan a los aparatos cardiovascular, respiratorio, digestivo, ocular, a las funciones motoras y en gran medida, se alteran las funciones psicológicas. El consumo crónico puede afectar a la descendencia y puede producir cuadros psiquiátricos.

\section{BIBLIOGRAFIA}

Adams IB, Martin BR. Cannabis: pharmacology and toxicology in animals and humans. Addiction 1996; 91: 1585-1614.

Benson MK, Bentley AM. Lung disease induced by drug addiction. Thorax 1995; 50: 1125-1127.

Brill H, Nahas, G.G. Cannabis intoxication and mental ilness. En: G.G. Nahas (ed): Marijuana in 
Science and Medicine. Nueva York: Raven. 1984.

García A, Mur A. Marihuana y gestación: repercusiones sobre la gestante, el feto y el recién nacido. Med Clin 1991, 96: 106-109.

Gold, M.S.Marihuana. Barcelona: Ediciones en Neurociencias. 1991.

Giannini, A.J.Marijuana and psychopathology. En: M.S. Gold y A.E. Slaby (eds). Dual diagnosis in substance abuse. Nueva York: Marcel Dekker, Inc. 1991.

Lorenzo P, Leza J C Utilidad terapéutica del cannabis y derivados. Adicciones 2000, vol 12, supl. 2.

Marselos M, Karamanakos P. Mutagenicity, Developmental toxicity and carcinogenicity of cannabis. Addiction Biol. 1999, 4: 5-12.

Millman, R.B, Bordwine, A. Cannabis. En: M. Galanter y H.D.Kleber (eds). Tratamiento de los trastornos por abuso de sutancias Barcelona: Masson. 1997. pp. 91-109.

Navarro M, Rubio P, de Fonseca FR. Behavioral consequences of maternal exposure to natural cannabinoids in rats. Psychopharmacology 1995, 122: 1-14.

Pertwee RG Pharmacology of Cannabinoid CB1 and CB2 receptors. Pharmacol Ther 1997, 74:129-180.

Quiroga M Cannabis efectos nocivos sobre la salud mental. Adicciones 2000, vol 12, supl. 2.

Tashlin DP, Calvarese BM, Simmons MS, Shapiro BJ. Respiratory status of seventy-four habitual marijuana smokers. Chest 1980, 78: 699-706.

Tennant FS, Preble M, Prendergast TJ, Ventry P. Medical manifestations associated with hashish. JAMA 1971; 216: 1965-1969. 